\title{
37. On the Continuous Cohomology of the Lie Algebra of Vector Fields
}

\author{
By Toru TsujIshita \\ Osaka University \\ (Communicated by Kunihiko KodaIra, M. J. A., Sept. 12, 1977)
}

$\S 1$. Let $M$ be a smooth manifold and $\mathcal{L}_{M}$ the topological Lie algebra of all smooth vector fields on $M$. Recently Haefliger ([4]) and G. Segal (unpublished) proved that the continuous cohomology $H\left(\mathcal{L}_{M}\right)$ of $\mathcal{L}_{M}$ with the trivial coefficient is isomorphic to the cohomology of the cross-section space of a canonically defined fibre bundle over $M$, as it was conjectured by Bott and Fuks.

As for the case associated with a non-trivial coefficient $\mathcal{A}$, Locik ([5][7]) has computed the cohomology of a certain subcomplex (called diagonal) of the standard cochain complex, and Reshetnikov ([9]) has announced partial results concerning the total continuous cohomology $H\left(\mathcal{L}_{M}, \mathcal{A}\right)$. In this note, we state a theorem which reduces the calculation of $H\left(\mathcal{L}_{M}, \mathcal{A}\right)$ to that of $H\left(\mathcal{L}_{M}\right)$ and the diagonal cohomology $H_{\Delta}\left(\mathcal{L}_{M}, \mathcal{A}\right)$ modulo the computation of differential torsion product. Thus the problem is reduced to a purely algebraic one. Details will be published elsewhere.

After the author had obtained the results (Theorems 1-3), he heard from Professor Fuks that they had been obtained by him and G. Segal in 1974, although they had never published them.

The author would like to express his gratitude to Professor Fuks, who taught him the neat consequence Theorem 4 of Theorem 3 and remarked that the case of the topological Lie algebra $\mathcal{L}_{M}^{c}$ of vector fields with compact supports can be treated similarly. The author would like to thank Professors A. Tsuchiya and S. Morita for their valuable helps in proving Theorem 2.

Section 2 recalls briefly the definition of Lie algebra cohomology and states the main theorem (Theorem 1 ) in the case $\mathcal{A}=C^{\infty}(M)$, elements of $\mathcal{L}_{M}$ acting on $C^{\infty}(M)$ as derivations. We state a topological interpretation of $H\left(\mathcal{L}_{M}, C^{\infty}(M)\right)$ in Section 3 and apply Theorem 1 to the particular cases $M=\boldsymbol{R}^{n}$ and $M=S^{1}$ in Section 4 . Section 5 states the main theorem in the general case. We consider the case of $\mathcal{L}_{M}^{c}$ in Section 6 .

§2. Let $\mathscr{V}$ be a topological $\mathcal{L}_{M}$-algebra. Let $C^{p}\left(\mathcal{L}_{M}, \mathscr{W}\right)(p>0)$ be the space of all continuous alternating multilinear mappings from $\mathcal{L}_{M} \times \cdots \times \mathcal{L}_{M}(p$ times $)$ to $\mathscr{W}$ and $C^{0}\left(\mathcal{L}_{M}, \mathscr{W}\right)=\mathscr{W}$. The differential 
$d \omega \in C^{p+1}\left(\mathcal{L}_{M}, \mathscr{W}\right)$ of $\omega \in C^{p}\left(\mathcal{L}_{M}, \mathscr{W}\right)$ and the product $\omega \eta \in C^{p+q}\left(\mathcal{L}_{M}, \mathscr{W}\right)$ of $\omega \in C^{p}\left(\mathcal{L}_{M}, \mathscr{W}\right)$ and $\eta \in C^{q}\left(\mathcal{L}_{M}, \mathscr{W}\right)$ are defined by the usual formulas. Then $C\left(\mathcal{L}_{M}, \mathscr{W}\right)=\left\{\oplus C^{p}\left(\mathcal{L}_{M}, \mathscr{W}\right), d\right\}$ turns out to be a commutative differential graded algebra (DG algebra for short). Its cohomology algebra will be denoted by $H\left(\mathcal{L}_{M}, \mathscr{W}\right)$.

Putting $\mathscr{W}=\boldsymbol{R}$ and $\mathscr{W}=C^{\infty}(M)$ with the usual $\mathcal{L}_{M}$-action and $C^{\infty}$ topology, we obtain DG algebras $C\left(\mathcal{L}_{M}, \boldsymbol{R}\right)$ and $C\left(\mathcal{L}_{M}, C^{\infty}(M)\right)$. Furthermore, put $C_{\Delta}^{0}\left(\mathcal{L}_{M}, C^{\infty}(M)\right)=C^{\infty}(M)$ and, for $p>0$,

$$
\begin{aligned}
C_{\downarrow}^{p}\left(\mathcal{L}_{M}, C^{\infty}(M)\right)= & \left\{\omega \in C^{p}\left(\mathcal{L}_{M}, C^{\infty}(M)\right) ; \operatorname{supp} \omega\left(X_{1}, \cdots, X_{p}\right)\right. \\
& \left.\subset \bigcap_{i=1}^{p} \operatorname{supp} X_{i}\left(X_{i} \in \mathcal{L}_{M}\right)\right\} .
\end{aligned}
$$

Then $C_{\Delta}\left(\mathcal{L}_{M}, C^{\infty}(M)\right)=\oplus C_{\Delta}^{p}\left(\mathcal{L}_{M}, C^{\infty}(M)\right)$ is a DG subalgebra of $C\left(\mathcal{L}_{M}\right.$, $C^{\infty}(M)$ ). We note that the de Rham DG algebra $\Omega_{M}$ of $M$ can be naturally identified with a DG subalgebra of $C_{\Delta}\left(\mathcal{L}_{M}, C^{\infty}(M)\right)$.

We shall denote by $\hat{C}\left(\mathcal{L}_{M}, \Omega_{M}\right)$ the completed tensor product of the DG algebras $C\left(\mathcal{L}_{M}, R\right)$ and $\Omega_{M}$ equipped with natural topologies. The underlying space of $\hat{C}\left(\mathcal{L}_{M}, \Omega_{M}\right)$ can be canonically identified with that of $C\left(\mathcal{L}_{M}, \Omega_{M}\right)$. Thus we can define as above a DG subalgebra $\hat{C}_{\Delta}\left(\mathcal{L}_{M}\right.$, $\left.\Omega_{M}\right)$ of $\hat{C}\left(\mathcal{L}_{M}, \Omega_{M}\right)$.

The inclusion mapping $\iota: R \subset C^{\infty}(M)$ being a continuous $\mathcal{L}_{M^{-}}$ homomorphism, there is a DG algebra homomorphism $\iota_{*}: C\left(\mathcal{L}_{M}, \boldsymbol{R}\right)$ $\rightarrow C\left(\mathcal{L}_{M}, C^{\infty}(M)\right)$. Consider $\kappa=\iota_{*} \hat{\otimes} j: \hat{C}\left(\mathcal{L}_{M}, \Omega_{M}\right)=C\left(\mathcal{L}_{M}, R\right) \hat{\otimes} \Omega_{M} \rightarrow C\left(\mathcal{L}_{M}\right.$, $\left.C^{\infty}(M)\right)$, where $j: \Omega_{M} \longrightarrow C_{\Delta}\left(\mathcal{L}_{M}, C^{\infty}(M)\right)$ is the natural inclusion mapping mentioned above. It is easy to see that $\hat{C}_{\Delta}\left(\mathcal{L}_{M}, \Omega_{M}\right)$ is mapped into $C_{\Delta}\left(\mathcal{L}_{M}, C^{\infty}(M)\right)$ by $\kappa$. Thus we get the following commutative diagram of DG algebra homomorphisms :

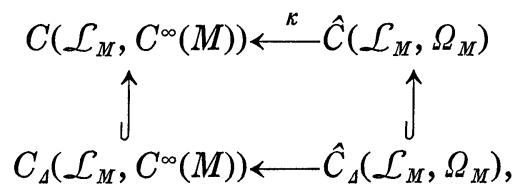

which induces a natural homomorphism of graded algebras:

$$
\alpha: \operatorname{Tor}_{\hat{C}_{\Delta}\left(\mathcal{L}_{M}, \Omega_{M}\right)}\left(\hat{C}\left(\mathcal{L}_{M}, \Omega_{M}\right), C_{\Delta}\left(\mathcal{L}_{M}, C^{\infty}(M)\right)\right) \rightarrow H\left(\mathcal{L}_{M}, C^{\infty}(M)\right),
$$

where Tor denotes the differential torsion product (cf. [2]).

By technical reasons, we shall assume from now throughout this paper that $M$ satisfies the following conditions:

$H^{*}(M, R)$ is finite-dimensional and for each $p(0 \leqq p \leqq \operatorname{dim} M)$, there is a closed subspace $X^{p}$ of $\Omega_{M}^{p}$ such that $X^{p} \oplus Z^{p} \cong \Omega_{M}^{p}$, where $Z^{p}$ is the subspace of all the closed $p$-forms.

Remark 1. It is well known that these conditions are satisfied by compact manifolds. Besides, it is easy to see that $\boldsymbol{R}^{n}$ satisfies them. It is probable that the former condition implies the latter. 
Theorem 1. $\alpha$ is an isomorphism.

By the general theory of differential torsion product (cf. [2]), we get the following

Corollary 1. There is a spectral sequence $E$ converging to $H\left(\mathcal{L}_{M}, C^{\infty}(M)\right)$ such that

$$
E_{2} \cong \operatorname{Tor}_{\hat{H}_{\Delta}\left(\mathcal{L}_{M}, \Omega_{M}\right)}\left(\hat{H}\left(\mathcal{L}_{M}, \Omega_{M}\right), H_{\Delta}\left(\mathcal{L}_{M}, C^{\infty}(M)\right)\right),
$$

where $\hat{H}_{\Delta}\left(\mathcal{L}_{M}, \Omega_{M}\right), \hat{H}\left(\mathcal{L}_{M}, \Omega_{M}\right)$ and $H_{\Delta}\left(\mathcal{L}_{M}, C^{\infty}(M)\right)$ denote the cohomology algebras of the $D G$ algebras $\hat{C}_{\Delta}\left(\mathcal{L}_{M}, \Omega_{M}\right), \hat{C}\left(\mathcal{L}_{M}, \Omega_{M}\right)$ and $C_{\Delta}\left(\mathcal{L}_{M}, C^{\infty}(M)\right)$ respectively and Tor denotes the torsion product of graded algebras.

Remark 2. The algebras $\hat{H}_{\Delta}\left(\mathcal{L}_{M}, \Omega_{M}\right), \hat{H}\left(\mathcal{L}_{M}, \Omega_{M}\right) \cong H\left(\mathcal{L}_{M}, \boldsymbol{R}\right)$ $\otimes H^{*}(M, R)$ and $H_{\Delta}\left(\mathcal{L}_{M}, C^{\infty}(M)\right)$ are known in principle (cf. [3]-[6]).

Remark 3. The computation of differential torsion product is difficult in general.

§3. From Theorem 1, we derive the following topological interpretation of the graded algebra $H\left(\mathcal{L}_{M}, C^{\infty}(M)\right)$.

Let $B$ be the principal $U(n)$-bundle associated to the complexification of the real tangent bundle of $M$ with a Riemannian metric, where $n=\operatorname{dim} M$. Let $\hat{E} U$ be the restriction of the universal principal $U(n)$ bundle to the $2 n$-skeleton of the base with respect to the usual cell decomposition. Put $E=B \underset{U(n)}{\times} \hat{E} U$. Fixing a fibre inclusion $U(n) \longrightarrow \hat{E} U$, we obtain an inclusion $i: B \hookrightarrow E$. Let $\Gamma(E)$ be the space of all continuous sections of the bundle $E \rightarrow M$ equipped with the compact open topology. Let $\varepsilon: M \times \Gamma(E) \rightarrow E$ be the evaluation mapping. Let

$$
Y=B \times(M \times \Gamma(E))=\{(b, x, s) \in B \times M \times \Gamma(E) ; i(b)=\varepsilon(x, s)\} .
$$

Then we have the following

Theorem 2. If $\pi_{1}(M)=0$, then

$$
H\left(\mathcal{L}_{M}, C^{\infty}(M)\right) \cong H^{*}(Y, R)
$$

as graded algebras.

$\S 4$. We apply Theorem 1 to particular cases.

Let $M=\boldsymbol{R}^{n}$. The results of [3], [4] and [5] imply that there is the following commutative diagram of DG algebra homomorphisms :

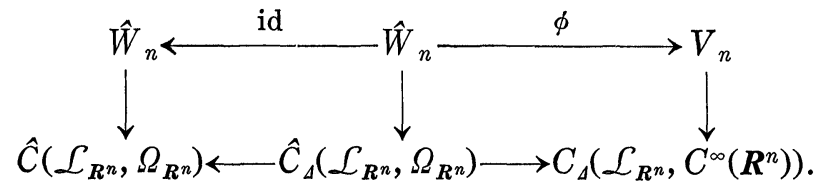

Here vertical homomorphisms induce isomorphisms between cohomology algebras; $V_{n}=S\left(\theta_{1}, \cdots, \theta_{n}\right)$ is the free trivial DG algebra generated by $\left\{\theta_{1}, \cdots, \theta_{n}\right\}$ with the gradation $\operatorname{deg} \theta_{i}=2 i-1 ; \hat{W}_{n}=W_{n} / I$, where $W_{n}=S\left(c_{1}, \cdots, c_{n}, \theta_{1}, \cdots, \theta_{n}\right)$ is the free DG algebra with $\operatorname{deg} c_{i}=2 i$, $\operatorname{deg} \theta_{i}=2 i-1, d \theta_{i}=c_{i}, d c_{i}=0$ and $I$ is the ideal generated by the monomials of $c_{i}$ 's of degree $>2 n ; \phi$ is defined by $\phi\left(c_{i}\right)=0, \phi\left(\theta_{i}\right)=\theta_{i}$. Then it follows 


$$
\begin{aligned}
& \operatorname{Tor}_{\hat{\sigma}_{\Delta}\left(\mathcal{L}_{\boldsymbol{R}^{n}, \Omega_{\left.\boldsymbol{R}^{n}\right)}}\right.}\left(\hat{C}\left(\mathcal{L}_{\boldsymbol{R}^{n}}, \Omega_{\boldsymbol{R}^{n}}\right), C_{\Delta}\left(\mathcal{L}_{\boldsymbol{R}^{n}}, C^{\infty}\left(\boldsymbol{R}^{n}\right)\right)\right) \\
& \cong \operatorname{Tor}_{\hat{W}_{n}}\left(\hat{W}_{n}, V_{n}\right) \cong V_{n}
\end{aligned}
$$

(cf. [2]). Hence by Remark 1 we obtain the following

Corollary 2. $H\left(\mathcal{L}_{\boldsymbol{R}^{n}}, C^{\infty}\left(\boldsymbol{R}^{n}\right)\right) \cong H_{\Delta}\left(\mathcal{L}_{\boldsymbol{R}^{n}}, C^{\infty}\left(\boldsymbol{R}^{n}\right)\right) \cong S\left(\theta_{1}, \cdots, \theta_{n}\right)$.

Let now $M=S^{1}$. Then we have the following commutative diagram of DG algebra homomorphisms :

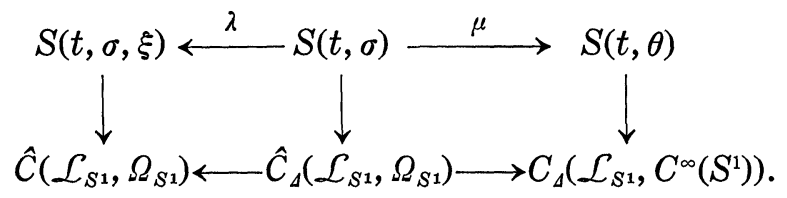

Here vertical homomorphisms induce isomorphisms between cohomology algebras, and $\operatorname{deg} t=\operatorname{deg} \theta=1, \operatorname{deg} \xi=2, \operatorname{deg} \sigma=3, d t=d \theta=d \sigma=d \xi$ $=0, \lambda(t)=t, \lambda(\sigma)=t \xi+\sigma, \mu(t)=t, \mu(\sigma)=0$. We can show that the triple (1) is equivalent to the following one:

$$
S(t, \sigma, \xi) \stackrel{\tilde{\lambda}}{\longleftarrow} S(t, \sigma) \stackrel{\mu}{\longrightarrow} S(t, \theta),
$$

where $\tilde{\lambda}(t)=t, \tilde{\lambda}(\sigma)=\sigma$. It is easy to see then that

$$
\operatorname{Tor}_{S(t, \sigma)}(S(t, \sigma, \xi), S(t, \theta)) \cong S(t, \theta, \xi) .
$$

Then we obtain the following

Corollary 3. $H\left(\mathcal{L}_{S^{1}}, C^{\infty}\left(S^{1}\right)\right) \cong S(t, \theta, \xi)$, where $\operatorname{deg} t=\operatorname{deg} \theta=1$, $\operatorname{deg} \xi=2$.

Remark 4. Reshetnikov proved Corollary 3 in [8] using a completely different method.

$\S 5$. We consider now the general case. We denote by $J^{k} M(k \geqq 1)$ the $k$-th frame bundle of $M$. $J^{k} M$ is a principal $G^{k}$-bundle, where $G^{k}$ is the Lie group of $k$-jets at the origin 0 of diffeomorphisms of $\boldsymbol{R}^{n}$ fixing 0 . Let $A$ be a finite-dimensional real $G^{k}$-module. Put $\alpha=J^{k} M \underset{G^{k}}{\times} A$. Then $\alpha$ is a Diff $(M)$-bundle over $M$, whence the space $\mathcal{A}_{M}$ of all smooth sections of $\alpha$ can be naturally regarded as a topological $\mathcal{L}_{M}$-module.

We can define $C\left(\mathcal{L}_{M}, \mathcal{A}_{M}\right), C_{\Delta}\left(\mathcal{L}_{M}, \mathcal{A}_{M}\right)$ and $H\left(\mathcal{L}_{M}, \mathcal{A}_{M}\right)$ just as in Section 2. The natural pairing $C^{\infty}(M) \times \mathcal{A}_{M} \rightarrow \mathcal{A}_{M}$ gives rise to a DG $C_{\Delta}\left(\mathcal{L}_{M}, C^{\infty}(M)\right.$ )-module structure on $C_{\Delta}\left(\mathcal{L}_{M}, \mathcal{A}_{M}\right)$. Using $\kappa \mid \hat{C}_{\Delta}\left(\mathcal{L}_{M}, \Omega_{M}\right)$, we regard $C_{\Delta}\left(\mathcal{L}_{M}, \mathcal{A}_{M}\right)$ as a $\mathrm{DG} \hat{C}_{\Delta}\left(\mathcal{L}_{M}, \Omega_{M}\right)$-module.

On the other hand, the $G^{k}$-module $A$ gives rise to a topological $L_{0^{-}}$ module $A$. Here $L_{0}$ denotes the topological Lie algebra of formal vector fields of $n$-variables without constant terms. Using the usual inclusion $\mathrm{gl}(n, \boldsymbol{R}) \smile L_{0}, A$ is also regarded as a $\mathfrak{g l}(n, \boldsymbol{R})$-module. We shall assume from now that $A$ is reductive as a gl $(n, R)$-module.

Theorem 3.

$$
H\left(\mathcal{L}_{M}, \mathcal{A}_{M}\right) \cong \operatorname{Tor}_{\hat{C}_{\Delta}\left(\mathcal{L}_{M}, \Omega_{M}\right)}\left(\hat{C}\left(\mathcal{L}_{M}, \Omega_{M}\right), C_{\Delta}\left(\mathcal{L}_{M}, \mathcal{A}_{M}\right)\right) .
$$

Theorem 3 and a straightforward generalization of the result of [7] to $L_{0}$-modules imply the following 


\section{Theorem 4.}

$$
H\left(\mathcal{L}_{M}, \mathcal{A}_{M}\right) \cong H\left(\mathcal{L}_{M}, C^{\infty}(M)\right) \otimes H\left(L_{0}, \mathfrak{g} \mathfrak{K}(n, R), A\right) .
$$

Remark 5. $H\left(L_{0}, \mathfrak{g} \mathfrak{l}(n, R), A\right)$ is the relative cohomology of $L_{0}$ with respect to the subalgebra $g \mathfrak{l}(n, R)$. It is unknown except for a few $G^{1-}$ modules $A$ (cf. [1][10]).

$\S 6$. Let $\mathcal{L}_{M}^{c}$ be the topological Lie algebra of all the smooth vector fields with compact supports. Let $\mathcal{A}_{M}$ be as in Section 5. Then $\mathcal{A}_{M}$ is also a topological $\mathcal{L}_{M}^{c}$-module. Thus we can define $C\left(\mathcal{L}_{M}^{c}, \mathcal{A}_{M}\right)$, $C_{\Delta}\left(\mathcal{L}_{M}^{c}, \mathcal{A}_{M}\right)$ and $H\left(\mathcal{L}_{M}^{c}, \mathcal{A}_{M}\right)$ just as before.

On the other hand, we can define $\hat{C}\left(\mathcal{L}_{M}^{c}, \Omega_{M}\right)$ and $\hat{C}_{\Delta}\left(\mathcal{L}_{M}^{c}, \Omega_{M}\right)$ in a natural way. Since $\hat{C}_{\Delta}\left(\mathcal{L}_{M}^{c}, \Omega_{M}\right)=\hat{C}_{\Delta}\left(\mathcal{L}_{M}, \Omega_{M}\right), \kappa$ induces again a DG $\hat{C}\left(\mathcal{L}_{M}^{c}, \Omega_{M}\right)$-module structure on $C_{\Delta}\left(\mathcal{L}_{M}^{c}, \mathcal{A}_{M}\right)$. We have then

Theorem 5.

$$
H\left(\mathcal{L}_{M}^{c}, \mathcal{A}_{M}\right) \cong \operatorname{Tor}_{\hat{C}_{\Delta}\left(\mathcal{L}_{M}^{c}, \Omega_{M}\right)}\left(\hat{C}\left(\mathcal{L}_{M}^{c}, \Omega_{M}\right), C_{\Delta}\left(\mathcal{L}_{M}^{c}, \mathcal{A}_{M}\right)\right) .
$$

Theorem 6.

$$
H\left(\mathcal{L}_{M}^{c}, \mathcal{A}_{M}\right) \cong H\left(\mathcal{L}_{M}^{c}, C^{\infty}(M)\right) \otimes H\left(L_{0}, \mathfrak{g l}(n, R), A\right) .
$$

\section{References}

[1] I. M. Gel'fand and D. B. Fuks: Cohomology of Lie algebra of vector fields with non-trivial coefficients. Funkt. Anal. i Ego Pril., 4(3), 10-25 (1970).

[2] V. K. A. M. Gugenheim and J. P. May: On the theory and applications of differential torsion products. Memoirs of AMS, 142 (1974).

[3] V. W. Guillemin: Cohomology of vector fields on a manifold. Advances in Math., 10, 192-220 (1973).

[4] A. Haefliger: Sur la cohomologie de l'algèbre de Lie des champs de vecteurs. Ann. scient. Éc. Norm. Sup., $4^{e}$ série, 9, 503-532 (1976).

[5] M. V. Losik: On the cohomologies of infinite-dimensional Lie algebras of vector fields. Funkt. Anal. i Ego Pril., 4(2), 43-53 (1970).

[6] - Cohomologies of Lie algebras of vector fields with coefficients in a trivial unitary representation. Funkt. Anal. i Ego Pril., 6(1), 24-36 (1972).

[7] —-: On cohomologies of Lie algebra of vector fields with non-trivial coefficients. Funkt. Anal. i Ego Pril., 6(4), 44-46 (1972).

[8] V. N. Reshetnikov: Cohomology of Lie algebras of vector fields on a manifold vanishing at a given point. Uspehi Mat. Nauk, 27 (1), 251-252 (1972).

[9] —-: On the cohomology of the Lie algebras of vector fields on a manifold with non-trivial coefficients. Dokl. Akad. Nauk SSSR, 208(5), 234-240 (1973).

[10] K. Shiga: Cohomology of Lie algebras over a manifold. I. J. Math. Soc. Japan, 26 (2), 324-361 (1974). 